\title{
Research Progress on Strengthen Mechanism and Technology of Mechanical Processing
}

\author{
Miao $\mathrm{He}^{1, \mathrm{a}}$, Yi Jiang ${ }^{2}$ \\ ${ }^{1,2}$ Nanchang Institute of Science and Technology, 330108 \\ ahemiaojx@126.com
}

Keywords: mechanical processing; strengthen mechanism; technology

\begin{abstract}
Along with the ongoing society development, people gradually gets a higher demand for daily life quality, which means a vital function for mechanical processing industry in its mechanism and technology development. This paper mainly talks about the development research on mechanism and technology in mechanical processing from current situation to future tendency.

Following the rapid society development speed, technology has already posed great influence on the traditional techniques which shows a steady developing trend in Chinese industry field. This raised the requirements for basic materials and components to adapt a more complicated circumstance in mechanical process environment, especially in their abrasion and corrosion performance. As a result, requirement for mechanical processing raised to a new level.
\end{abstract}

\section{Strengthen Mechanism in Mechanical Processing}

From microcosmic aspect, the closer of atomicity among materials is, the higher intensity of the material is. Then the plastic deformation of materials can be completed by slip. With the slip force, critical profit can be found out. However, the actual practice intensity of materials is lower than the calculated actual intensity by critical stress. By this situation, the crystal position error theory refers to the calculated actual intensity. Crystal position error theory means that the plastic deformation of materials is realized by dislocation movements of crystals deeply showing the use of atoms. Results comes at a different place from the material intensity, but still proves the strengthen mechanism in materials. As for the real production process, expansion of crystal dislocation density in materials can block the crystal movements forming a dislocation movement. According to different kinds of materials and strengthen mechanism in actual production and different processing methods in its equipment and rolling technology and process, strengthen mechanism can be divided into irradiation strengthening, solid solution strengthening and directional phase strengthening three types.

First, the realization of dislocation strengthening. In the real production process, the critical shear stress of crystal dislocation is lower than the theoretical value. Dislocation intensity of materials intensifies by the interaction in crystals. These phenomenons makes it harder to increase dislocation movements and raises the material intensity and stiffness. Things will become more complicated in the real production for the fact that even with the simulation model, it would only be used for describing the dislocation movements in metal intensity. On the strengthen mechanism in dislocation, the flowing stress on the slip plane in the material production can get sufficient limitation and stress within minutes. Scanning the distance between them can get the production data. The data should be same with the resistance force on dislocation slip plane. Again with the dislocation strengthen, the flowing stress would be separated into grid resistance and dislocation strengthening.

Second, grain boundary strengthening in mechanical processing. Grain boundary strengthening obstacles will pose influence to materials to some extent. During the transformation, dislocation movements will produce resistance force and it will gradually increase at the time grain boundary turning into dislocation sources, which increases the material intensity. This will exert both direct and indirect influence. Crystal resistors in grain boundary sliding will leads to direct reinforcement exercising an obvious dislocation obstruction and higher deformation from more concentrated stress 
so that it effects the crystal effect. On the indirect influence, otherwise, compatibility of flexibility and deformation between crystals will change and form higher stress concentration in crystals and sliding in order to improve the crystal intensity. Graph below shows the intensity distribution inside the crystal:

Third, strain strengthening in the strengthen mechanism. During the transformation period, strain hardening will exert several extra dislocation to the material and form an interaction in the dislocation movements to set up objections, in this way enhanced the material intensity. Strain strengthening is also called work hardening including three stages: easy sliding stage, linear hardening stage and dynamic recovery hardening stage. In the easy sliding stage, the work hardening rate will drop continuously affected by crystal structure, its orientation and impurities within the material. The work hardening rate will go upwards in the linear hardening stage. The behavior of materials can be approximated by a linear curve. The hardening rate in this stage can not be affected by compounds and crystal orientation easily. In the last stage, hardening rate drops with a parabolic curve of material behaviors. Reaction rate, stacking fault energy and strain rate effect of the transformation temperature shows little changes in the stress transformation process and ends together with the material interest saturation point. The following graph shows the single crystal stress-strain curve.

Fourth, preferred orientation strengthening in strengthen mechanism. Six preferred material orientation during the material processing probably leads to deformation of crystal intensity in the improving plastic material process. This kind of strengthen mechanism will results to fabric texture and rolling texture. Materials possess the distortion uniformity and its energy always lies in a linear structure. Among this, deformable texture model is mainly influenced by total and half constraint. With the deformable texture increasing, material components, structures, temperature effects and technology elements will change together. In the practical production, when the distortion temperature raises, the crystal gets bigger, which leads to unstable changes inside and lower the intensity. The following graph is about the changing curve of flowing stress in the temperature-dislocation strengthening function.

\section{Technology in Mechanical Processing}

Firstly, shot blasting treatment. This treatment will be performed in a total manly-controlled situation. This shot blasting treatment means spray many projectiles onto the material surface to promote a plastic transformation and refining the crystals. In this way realizing an ideal structure and working with the residual stress to refine its intensity and extend its service life. The most frequently used place for this treatment is the surface strengthening part. The advantages are high efficient, simple operation, low production cost and improve material surface stiffness and service life. Material stress corrosion crack performance will be improved as long as with the shot blasting technique. For materials with alternating stress and stress corrosion qualities, spray coating will be the best. The following picture shows the specific shot blasting technique.

Second, laser shock processing. This is a new type of shot blasting technique. However, the divergence of equipment and technology attribute this new type to another material treatment. Laser shock processing normally uses short-pulse laser of high power density and strongly constrained layer to penetrate materials. Then the surface will absorb the laser energy into vaporization form. Steams from the vaporization will continue to absorb the laser energy and explode with plasma. Substances are controlled under the surface pushing the pressure into strong stress waves. When the pressure reaches a high degree, the material will receive a violent plastic transformation leading to an intensive increase to the ground intensity and crystal refinement. This hence improves the surface stiffness and rheological strength.

Thirdly, rolling strengthening. This technique uses elastic metal composite to bring bear on rolling material appearances to change the plastic transformation of composition structure and physical properties, shape, size and mechanical properties. During the rolling strengthening process, 
the material magnitude and laser shaping intensity can be improved. Rolling outside treatment can replace some materials and promote the finishing machining materials in the real production. Outside processing of compression rollers can leads to elastic deformation and plastic deformation. If the rolling pressure rolling strengthening technique surpasses the limited yield point, crystals will slip, dislocate, break and plastic deform. Outside back to its formal elastic deformation and exert influence to plastic deformation improving the material magnitude. The following picture explains the rolling strengthening.

\section{Summary}

Strengthen the materials in mechanical processing can make a great progress in the material quality, magnitude and corrosion resistance. China should and will invest more talents, scientific sources and efforts in this direction to bring about a more satisfied performance of mechanical processing. Believing in their continuous efforts, we can expect an era of mechanical process with more effective solutions and techniques.

\section{Reference}

[1] Zhanqiang Liu, Meng He, Jian Zhao. Research Progress in Strengthen Mechanism and Technology of Mechanical Processing[J]. China Mechanical Engineering, 2015, 03:403-413.

[2] Haohu Yang. Analyze on Strengthen Mechanism and Technology of Mechanical Processing[J]. Hunan Agricultural, 2016,07:6-7.

[3] Dandan Yuan. Research on Material Removal Mechanism of Polymethyl Methacrylate Nano Machine Process[D]. Tianjin University, 2014.

[4] Yiding Cao, Mingdao Xin, Huande Xie. Analyze and Experiments of Outside Boiling Heat Transfer on T-Structure Mechanical Processing Strengthen[J]. Journal of Engineering Thermophysics, 1986, 01:63-66. 